\title{
Hot Hypernuclear Matter in the Modified Quark Meson Coupling Model
}

\author{
I. Zakout ${ }^{1,2}$, H. R. Jaqaman ${ }^{1 *}$, S. Pal ${ }^{2}$, H. Stöcker ${ }^{2}$ and W. Greiner ${ }^{2}$ \\ 1 Department of Physics, Bethlehem University, P.O. Box 9, Bethlehem, Palestine \\ ${ }^{2}$ Institut für Theoretische Physik, J. W. Goethe Universität, Robert Mayer Staße 8-10, Postfach \\ 11 1932, D-60054, Frankfurt am Main, Germany
}

(February 22, 1999)

\begin{abstract}
Hot hypernuclear matter is investigated in an explicit $S U(3)$ quark model based on a mean field description of nonoverlapping baryon bags bound by the self-consistent exchange of scalar $\sigma, \zeta$ and vector $\omega, \phi$ mesons. The $\sigma, \omega$ mean fields are assumed to couple to the $u, d$-quarks while the $\zeta, \phi$ mean fields are coupled to the $s$-quark. The coupling constants of the mean fields with the quarks are assumed to satisfy $S U(6)$ symmetry. The calculations take into account the medium dependence of the bag parameter on the scalar fields $\sigma, \zeta$. We consider only the octet baryons $N, \Lambda, \Sigma, \Xi$ in hypernuclear matter. An ideal gas of the strange mesons $K$ and $K^{*}$ is introduced to keep zero net strangeness density. Our results for symmetric hypernuclear matter show that a phase transition takes place at a critical temperature around $180 \mathrm{MeV}$ in which the scalar mean fields $\sigma, \zeta$ take nonzero values at zero baryon density. Furthermore, the bag contants of the baryons decrease significantly at and above this critical temperature indicating the onset of quark deconfinement. The present results imply that the onset of quark deconfinement in $S U(3)$ hypernuclear matter is much stronger than in $S U(2)$ nuclear matter.
\end{abstract}

PACS:21.65.+f, 24.85.+p, 12.39Ba

*hjaqaman@bethlehem.edu 


\section{INTRODUCTION}

The majority of nuclear phenomena have been successfully described in relativistic meanfield theory using only hadronic degrees of freedom [1] [3]. However, due to the observations which revealed the medium modification of the internal structure of the baryons 何, it has become essential to explicitly incorporate the quark-gluon degrees of freedom while respecting the established model based on the hadronic degrees of freedom in nuclei. One of the first models put forward along these lines is the quark-meson coupling (QMC) model, proposed by Guichon [5], which describes nuclear matter as a collection of non-overlapping MIT bags interacting through the self-consistent exchange of scalar $\sigma$ and vector $\omega$ mesons in the mean field approximation with the meson fields directly coupled to the quarks [6,7]. The scalar $\sigma$ meson is supposed to simulate the exchange of correlated pairs of pions and may represent a very broad resonance observed in $\pi \pi$ scattering, while the vector $\omega$ meson is identified with the actual meson having a mass of about $780 \mathrm{MeV}$. In the chiral models, the scalar $\sigma$ and vetcor $\omega$ mean fields represent the $u, d$ quark condensates [8,9]. The QMC model thus incorporates explicitly the quark degrees of freedom and this has nontrivial consequences [6.7]. It also have been extended to study superheavy finite nuclei [10,11].

In the so-called modified quark meson coupling model (MQMC) [12,13], it has been further suggested that including a medium-dependent bag parameter may be essential for the success of relativistic nuclear phenomenology. It was found that when the bag parameter is significantly reduced in the nuclear medium with respect to its free-space value, large cancelling isoscalar Lorentz scalar and vector potentials for the nucleon in nuclear matter emerge naturally. Such potentials are comparable to those suggested by relativistic nuclear phenomenology and finite density QCD sum rules [14]. The density-dependence of the bag parameter is introduced by coupling it to the scalar meson field [12]. This coupling is motivated by invoking the nontopological soliton model for the nucleon [15. In this model a scalar soliton field provides the confinement of the quarks. This effect of the soliton field is, roughly speaking, mimicked by the introduction of the bag parameter in the Bag Model. When a nucleon soliton is placed in a nuclear environment, the scalar soliton field interacts with the scalar mean field. It is thus reasonable to couple the bag parameter to the scalar mean fields 12,13.

The QMC model was extended to finite temperatures to investigate the liquid-gas phase transition in nuclear matter [16]. Recently, the MQMC model has been also extended to finite temperature [17 19] and has been applied to the study of the properties of nuclear matter where it was found that the bag parameter decreases appreciably above a critical temperature $T_{c} \approx 200 \mathrm{MeV}$ indicating the onset of quark deconfinement 18. The effect of glueball exchange as well as a realization of the broken scale invariance of quantum chromodynamics has also been investigated in the MQMC model through the introduction of a dilaton field [19]. It was found that the introduction of the dilaton potential improves the shape of the saturation curve at $\mathrm{T}=0$ and affects hot nuclear matter significantly 19 .

In the present work, we extend the MQMC model to hot hypernuclear matter by introducing the scalar $\zeta$ and vector $\phi$ mean fields that are coupled to the $s$-quark in addition to the $\sigma$ and $\omega$ fields which are coupled to the $u, d$-quarks. The $\zeta$ and $\phi$ fields are identified with the real mesons having masses $m_{\zeta}=975$ and $m_{\phi}=1020 \mathrm{MeV}$ respectively [8,9, 20]. Hy-

pernuclear matter is considered to contain the octet $p, n, \Lambda, \Sigma^{+}, \Sigma^{0}, \Sigma^{-}, \Xi^{0}$ and $\Xi^{-}$baryons 
[21]. We introduce an ideal gas of kaons to keep zero net strangeness density $\rho_{S}=0$. We simplify the calculations by considering symmetric hypernuclear matter whereby the octet baryons reduce to 4 species: $2 N, \Lambda, 3 \Sigma, 2 \Xi$.

The outline of the paper is as follows. In section II, we present the MQMC model for hypernuclear matter at finite temperature, together with the details of the self-consistency conditions for the vector and scalar mean fields. In section III, we discuss our results and present our conclusions.

\section{THE QUARK MESON COUPLING MODEL FOR HYPERNUCLEAR MATTER}

The quark field $\psi_{q}(\vec{r}, t)$ inside a bag of radius $R_{i}$ representing a baryon of species $i$ satisfies the Dirac equation

$$
\left[i \gamma^{\mu} \partial_{\mu}-m_{q}^{0}+\left(g_{\sigma}^{q} \sigma-g_{\omega}^{q} \omega_{\mu} \gamma^{\mu}\right) \delta_{q r}+\left(g_{\zeta}^{q} \zeta-g_{\phi}^{q} \phi_{\mu} \gamma^{\mu}\right) \delta_{q s}\right] \psi_{q}(\vec{r}, t)=0 .
$$

where $m_{q}^{0}$ is the current mass of the quarks of flavor $q$ and where $r$ refers to the up or down quarks and $s$ refers to the strange quark. The Kronecker deltas insure that the $\mathrm{u}$, d quarks are coupled only to the $\sigma$ and $\omega$ fields while the s-quark is coupled only to the $\zeta$ and $\phi$ fields. In the mean field approximation all the meson fields are treated classically and, for nuclear matter in this approximation, these fields are translationally invariant. Moreover, because of rotational invariance, the space-like components of the vector fields vanish so that we have $\omega_{\mu} \gamma^{\mu}=<\omega_{0}>\gamma^{0}=\omega \gamma^{0}$ and $\phi_{\mu} \gamma^{\mu}=<\phi_{0}>\gamma^{0}=\phi \gamma^{0}$.

The single-particle quark and antiquark energies in units of $R_{i}^{-1}$ for quark flavor $q$ are given by

$$
\epsilon_{q_{ \pm}}^{n \kappa}=\Omega_{q}^{n \kappa} \pm\left(g_{\omega}^{q} \omega R_{i} \delta_{q r}+g_{\phi}^{q} \phi R_{i} \delta_{q s}\right)
$$

where

$$
\Omega_{q}^{n \kappa}=\sqrt{x_{n \kappa}^{q}{ }^{2}+R_{i}^{2} m_{q}^{* 2}}
$$

and

$$
m_{q}^{*}=m_{q}^{0}-g_{\sigma}^{q} \sigma \delta_{q r}-g_{\zeta}^{q} \zeta \delta_{q s}
$$

are the effective quark kinetic energy and effective quark mass, respectively. The boundary condition for each quark of flavor $q$ at the bag surface is given by

$$
i \gamma \cdot \hat{n} \psi_{q}^{n \kappa}\left(x_{n \kappa}^{q}\right)=\psi_{q}^{n \kappa}\left(x_{n \kappa}^{q}\right),
$$

which determines the quark momentum $x_{n \kappa}^{q}$ in the state characterized by specific values of $n$ and $\kappa$. For a given value of the bag radius $R_{i}$ for baryon species $i$ and the scalar fields $\sigma$ and $\zeta$, the quark momentum $x_{n \kappa}^{q}$ is determined by the boundary condition Eq. (5) which, for quarks of flavor $q$ in a spherical bag, reduces to $j_{0}\left(x_{n \kappa}^{q}\right)=\beta_{q} j_{1}\left(x_{n \kappa}^{q}\right)$, where

$$
\beta_{q}=\sqrt{\frac{\Omega_{q}^{* n \kappa}(\sigma, \zeta)-R_{i} m_{q}^{*}(\sigma, \zeta)}{\Omega_{q}^{* n \kappa}(\sigma, \zeta)+R_{i} m_{q}^{*}(\sigma, \zeta)} .}
$$


The quark chemical potential $\mu_{q}$, assuming that there are three quarks in the baryon bag, is determined from $3=\sum_{q} n_{q}$ where $n_{q}$ is the number of quarks of flavor $\mathrm{q}$ and is determined by [17 19]

$$
n_{q}=\sum_{n \kappa}\left[\frac{1}{e^{\left(\epsilon_{q_{+}}^{n \kappa} / R-\mu_{q}\right) / T}+1}-\frac{1}{e^{\left(\epsilon_{q}^{n \kappa} / R+\mu_{q}\right) / T}+1}\right]
$$

The total energy from the quarks and antiquarks of each baryon of species $i$ is

$$
E_{\text {tot }}^{i}=\sum_{n \kappa q} n_{q} \frac{\Omega_{q}^{n \kappa}}{R}\left[\frac{1}{e^{\left(\epsilon_{q+}^{n \kappa} / R_{i}-\mu_{q}\right) / T}+1}+\frac{1}{e^{\left(\epsilon_{q-}^{n \kappa} / R_{i}+\mu_{q}\right) / T}+1}\right] .
$$

The bag energy for baryon species $i$ is given by

$$
E_{b a g}^{i}=E_{t o t}^{i}-\frac{Z_{i}}{R_{i}}+\frac{4 \pi}{3} R_{i}^{3} B_{i}(\sigma, \zeta)
$$

where $B_{i}=B_{i}(\sigma, \zeta)$ is the bag parameter. In the simple QMC model, the bag parameter $B$ is taken as $B_{0}$ corresponding to its value for a free baryon. The medium effects are taken into account in the MQMC model [12, 13] by coupling the bag parameter to the scalar meson fields. In the present work we generalize the coupling suggested in the latter references to the case of two scalar meson fields by using the following ansatz for the bag parameter

$$
B_{i}=B_{0} \exp \left[-\frac{4}{3} \frac{\left(\left(n_{u}+n_{d}\right) g_{\sigma}^{B} \sigma+n_{s} g_{\zeta}^{B} \zeta\right)}{M_{i}}\right]
$$

with $g_{\sigma}^{B}$ and $g_{\zeta}^{B}$ as additional parameters. The spurious center-of-mass momentum in the bag is subtracted to obtain the effective baryon mass 22

$$
M_{i}^{*}=\sqrt{E_{b a g}^{i}{ }^{2}-<p_{c m}^{2}>^{i}}
$$

where

$$
<p_{c m}^{2}>^{i}=\frac{<x^{2}>^{i}}{R_{i}^{2}}
$$

and

$$
<x^{2}>^{i}=\sum_{n \kappa q} n_{q} x_{n \kappa}^{q 2}\left[\frac{1}{e^{\left(\epsilon_{+}^{n \kappa} / R_{i}-\mu_{q}\right) / T}+1}+\frac{1}{e^{\left(\epsilon_{q_{-} \kappa}^{n \kappa} / R_{i}+\mu_{q}\right) / T}+1}\right] .
$$

The bag radius $R_{i}$ for baryon species $i$ is obtained through the minimization of the baryon mass with respect to the bag radius [7, 12, 17 19

$$
\frac{\partial M_{i}^{*}}{\partial R_{i}}=0
$$

The total energy density at finite temperature $T$ and at finite baryon density $\rho_{B}$ reads 


$$
\begin{aligned}
\varepsilon & =\sum_{i}^{\text {Baryons }} \frac{\gamma_{i}}{(2 \pi)^{3}} \int d^{3} k \sqrt{k^{2}+M_{i}^{* 2}}\left(f_{i}+\bar{f}_{i}\right)+\frac{1}{2} m_{\omega}^{2} \omega^{2}+\frac{1}{2} m_{\phi}^{2} \phi^{2}+\frac{1}{2} m_{\sigma}^{2} \sigma^{2}+\frac{1}{2} m_{\zeta}^{2} \zeta^{2} \\
& +\varepsilon_{K}^{i d}
\end{aligned}
$$

where $\gamma_{i}$ is the spin-isospin degeneracy factor of baryon species $i$ and where the last term corresponds to the energy density of the $K$-mesons treated here as an ideal gas. In Eq. (15) $f_{i}$ and $\bar{f}_{i}$ are the Fermi-Dirac distribution functions for the baryons and antibaryons of species $i$,

$$
f_{i}=\frac{1}{e^{\left(\epsilon_{i}^{*}-\mu_{i}^{*}\right) / T}+1}
$$

and

$$
\bar{f}_{i}=\frac{1}{e^{\left(\epsilon_{i}^{*}+\mu_{i}^{*}\right) / T}+1}
$$

where $\epsilon_{i}^{*}$ and $\mu_{i}^{*}$ are, respectively, the effective energy and effective chemical potential of baryon species $i$. These are given by $\epsilon_{i}^{*}=\sqrt{k^{2}+M_{i}^{* 2}}$ and $\mu_{i}^{*}=B_{i} \mu_{B}+S_{i} \mu_{S}-\left(g_{\omega i} \omega+g_{\phi i} \phi\right)$ where $B_{i}$ and $S_{i}$ are the baryon and strangeness quantum numbers and where $g_{\omega i}=$ $\left(n_{u}+n_{d}\right) g_{\omega i}^{q}$ and $g_{\phi i}=n_{s} g_{\phi i}^{q}$. The chemical potentials $\mu_{B}, \mu_{S}$ are determined by the self-consistency equations for the total baryonic density

$$
\rho_{B}=\frac{1}{(2 \pi)^{3}} \sum_{i} B_{i} \gamma_{i} \int d^{3} k\left(f_{i}-\bar{f}_{i}\right)
$$

and the total strangeness density

$$
\rho_{S}=\frac{1}{(2 \pi)^{3}} \sum_{i}^{\text {Baryons }} S_{i} \gamma_{i} \int d^{3} k\left(f_{i}-\bar{f}_{i}\right)+\sum_{i}^{\text {Kaons }} S_{i} \rho_{K i}^{i d}=0
$$

where in the last equation we have introduced the contribution of an ideal gas of $K$ and $K^{*}$ mesons to make the total strangeness density vanish identically. The vector mean fields are determined by

$$
\omega=\sum_{i} \frac{g_{\omega i}}{m_{\omega}^{2}} B_{i} \rho_{i}
$$

and

$$
\phi=\sum_{i} \frac{g_{\phi i}}{m_{\phi}^{2}} B_{i} \rho_{i}
$$

The pressure is the negative of the grand thermodynamic potential density and is given by

$$
P=\frac{1}{3} \sum_{i} \frac{\gamma_{i}}{(2 \pi)^{3}} \int d^{3} k \frac{k^{2}}{\epsilon_{i}^{*}}\left(f_{i}+\bar{f}_{i}\right)+\frac{1}{2} m_{\omega}^{2} \omega^{2}+\frac{1}{2} m_{\phi}^{2} \phi^{2}-\frac{1}{2} m_{\sigma}^{2} \sigma^{2}-\frac{1}{2} m_{\zeta}^{2} \zeta^{2}+P_{K}^{i d},
$$

where the summation $i$ runs over the 8 species of the baryon octet which reduces for symmetric hypernuclear matter to 4 species with $\gamma_{i}=4,2,6$ and 4 for $N, \Lambda, \Sigma$ and $\Xi$, respectively. 
In Eq. (22) $P_{K}^{i d}$ is the pressure of the ideal gas of $K$ mesons. The density of the $K$-mesons of species $i$ is given by

$$
\rho_{K i}^{i d}=\frac{\gamma_{i}^{K}}{(2 \pi)^{3}} \int d^{3} k\left(b_{i}-\bar{b}_{i}\right)
$$

where the spin-isospin degeneracy $\gamma_{i}^{K}=4,6$ for $K, K^{*}$, respectively. The Bose-Einstein distribution functions for the $K$ mesons are given by

$$
b_{i}=\frac{1}{e^{\left[\sqrt{k^{2}+M_{i}^{2}}-\mu_{i}\right] / T}-1}
$$

and

$$
\bar{b}_{i}=\frac{1}{e^{\left[\sqrt{k^{2}+M_{i}^{2}}+\mu_{i}\right] / T}-1}
$$

where $\mu_{i}=S_{i} \mu_{S}$ is the chemical potential of $K$-meson of species $i$. The total energy density and total pressure of the $K$-meson ideal gas are given by

$$
\varepsilon_{K}^{i d}=\sum_{i} \frac{\gamma_{i}^{K}}{(2 \pi)^{3}} \int d^{3} k \sqrt{k^{2}+M_{i}^{2}}\left(b_{i}-\bar{b}_{i}\right),
$$

and

$$
P_{K}^{i d}=\frac{1}{3} \sum_{i} \frac{\gamma_{i}^{K}}{(2 \pi)^{3}} \int d^{3} k \frac{k^{2}}{\sqrt{k^{2}+M_{i}^{2}}}\left(b_{i}-\bar{b}_{i}\right),
$$

respectively.

The scalar mean fields $\sigma$ and $\zeta$ are determined through the minimization of the thermodynamic potential or the maximizing of the pressure with respect to these fields. The pressure depends explicitly on the scalar mean fields through the $\frac{1}{2} m_{\sigma}^{2} \sigma^{2}$ and $\frac{1}{2} m_{\zeta}^{2} \zeta^{2}$ terms in Eq. (22). It also depends on the baryon effective masses $M_{i}^{*}$ which in turn also depend on $\sigma$ and $\zeta$. If we write the pressure as a function of $M_{i}^{*}, \sigma$ and $\zeta[18,19]$, the extremization of $P\left(M_{i}^{*}, \sigma, \zeta\right)$ with respect to the scalar mean field $\sigma$ can be written as

$$
\left(\frac{\partial P}{\partial \sigma}\right)_{\zeta}=\sum_{i}\left(\frac{\partial P}{\partial M_{i}^{*}}\right)_{\mu_{B}, T}\left(\frac{\partial M_{i}^{*}}{\partial \sigma}\right)_{\zeta}+\left(\frac{\partial P}{\partial \sigma}\right)_{\left\{M_{i}^{*}\right\}}=0
$$

where

$$
\left(\frac{\partial P}{\partial \sigma}\right)_{\left\{M_{i}^{*}\right\}}=-m_{\sigma}^{2} \sigma
$$

with a similar experssion for the extremization of $P\left(M_{i}^{*}, \sigma, \zeta\right)$ with respect to the scalar mean field $\zeta$. The derivative of the pressure with respect to effective mass $M_{i}^{*}$ reads 


$$
\begin{aligned}
\left(\frac{\partial P}{\partial M_{i}^{*}}\right)_{\mu_{i}, T}= & -\frac{1}{3} \frac{\gamma_{i}}{(2 \pi)^{3}} \int d^{3} k \frac{k^{2}}{\epsilon^{* 2}} \frac{M_{i}^{*}}{\epsilon_{i}^{*}}\left[f_{i}+\bar{f}_{i}\right] \\
& -\frac{1}{3} \frac{\gamma_{i}}{(2 \pi)^{3}} \frac{1}{T} \int d^{3} k \frac{k^{2}}{\epsilon_{i}^{*}} \frac{M_{i}^{*}}{\epsilon_{i}^{*}}\left[f_{i}\left(1-f_{i}\right)+\bar{f}_{i}\left(1-\bar{f}_{i}\right)\right] \\
& -\frac{1}{3} \frac{\gamma_{i}}{(2 \pi)^{3}} \frac{1}{T} g_{\omega i}\left(\frac{\partial \omega}{\partial M_{i}^{*}}\right)_{\mu_{i}, T} \int d^{3} k \frac{k^{2}}{\epsilon_{i}^{*}}\left[f_{i}\left(1-f_{i}\right)-\bar{f}_{i}\left(1-\bar{f}_{i}\right)\right] \\
& -\frac{1}{3} \frac{\gamma_{i}}{(2 \pi)^{3}} \frac{1}{T} g_{\phi i}\left(\frac{\partial \phi}{\partial M_{i}^{*}}\right)_{\mu_{i}, T} \int d^{3} k \frac{k^{2}}{\epsilon_{i}^{*}}\left[f_{i}\left(1-f_{i}\right)-\bar{f}_{i}\left(1-\bar{f}_{i}\right)\right] \\
& +m_{\omega}^{2} \omega\left(\frac{\partial \omega}{\partial M_{i}^{*}}\right)_{\mu_{i}, T} \\
& +m_{\phi}^{2} \phi\left(\frac{\partial \phi}{\partial M_{i}^{*}}\right)_{\mu_{i}, T} .
\end{aligned}
$$

Since the baryon chemical potential $\mu_{B}$ and temperature are treated as input parameters, the variation of the vector mean field $\omega$ with respect to the effective baryon mass $M_{i}^{*}$ at a given value of the baryon density $\rho_{B}$ reads

$$
\left(\frac{\partial \omega}{\partial M_{i}^{*}}\right)_{\mu_{i}, T}=-\frac{\left[g_{\omega i} / m_{\omega}^{2}\right]\left[\gamma_{i} /(2 \pi)^{3}\right][1 / T] \int d^{3} k \frac{M_{i}^{*}}{\epsilon_{i}^{*}}\left[f_{i}\left(1-f_{i}\right)-\bar{f}_{i}\left(1-\bar{f}_{i}\right)\right]}{1+\sum_{j}\left[g_{\omega j}^{2} / m_{\omega}^{2}\right]\left[\gamma_{j} /(2 \pi)^{3}\right][1 / T] \int d^{3} k\left[f_{j}\left(1-f_{j}\right)+\bar{f}_{j}\left(1-\bar{f}_{j}\right)\right]} .
$$

with a similar expression for $\left(\partial \phi / \partial M_{i}^{*}\right)_{\mu_{i}, T}$. The coupling of the scalar mean fields $\sigma$ and $\zeta$ with the quarks in the non-overlapping MIT bags through the solution of the point like Dirac equation should satisfy the self-consistency condition. These constraints are essential to obtain the correct solution of the scalar mean fields $\sigma$ and $\zeta$.

\section{RESULTS AND DISCUSSIONS}

We have studied hypernuclear matter at finite temperature using the modified quark meson coupling model which takes the medium-dependence of the bag into account. We choose a direct coupling of the bag parameter to the scalar mean fields $\sigma$ and $\zeta$ in the form given in Eq. (10). The bag parameter is taken as that adopted by Jin and Jennings [12] $B_{0}^{1 / 4}=188.1 \mathrm{MeV}$ and the free nucleon bag radius $R_{0}=0.60 \mathrm{fm}$. We have taken the current quark masses to be $m_{u}=m_{d}=0$ and $m_{s}=150 \mathrm{MeV}$. For $g_{\sigma}^{q}=1$, the values of the vector meson coupling constant and the parameter $g_{\sigma}^{B}$, as fitted from the saturation properties of nuclear matter, are given as $g_{\omega}^{2} / 4 \pi=\left(3 g_{\omega}^{q}\right)^{2} / 4 \pi=5.24$ and $g_{\sigma}^{B^{2}} / 4 \pi=\left(3 g_{\sigma}^{B^{q}}\right)^{2} / 4 \pi=3.69$. The $Z_{i}=2.03,1.814,1.629$ and 1.505 are chosen to reproduce the baryon masses at their experimental values $M_{N, \Lambda, \Sigma, \Xi}=939,1157,1193$, and $1313 \mathrm{MeV}$ respectively. Normal nuclear matter saturation density is taken as $\rho_{B 0}=0.17 \mathrm{fm}^{-3}$. The extra coupling constants needed to couple the scalar and vector mean fields $\zeta$ and $\phi$ to the $s$-quark are chosen to satisfy $S U(6)$ symmetry where $\left|g_{\zeta}^{q}\right|=\sqrt{2}\left|g_{\sigma}^{q}\right|$ and $\left|g_{\phi}^{q}\right|=\sqrt{2}\left|g_{\omega}^{q}\right|$ and $\left|g_{\zeta}^{B q}\right|=\sqrt{2}\left|g_{\sigma}^{B q}\right|$. If it is assumed that the mean fields $\zeta$ and $\phi$ are positive definite, then all the coupling constants are positive and the absolute value signs become redundant. 
The $\sigma$ mean field is supposed to simulate the exchange of correlated pairs of pions and may represent a very broad resonances observed in $\pi \pi$ scattering and fixed at $m_{\sigma}=550 \mathrm{MeV}$, while the vector $\omega$ meson is identified with the actual meson whose mass is $m_{\omega}=783 \mathrm{MeV}$. Since the mean fields, $\sigma$ and $\omega$, are considered as $\langle u \bar{d}>$ condensates, they interact only with $u, d$-quark in the baryons. On the other hand, the scalar and vector mean fields $\zeta, \phi$ are considered as actual mesons with $m_{\zeta}=975 \mathrm{MeV}$ and $m_{\phi}=1020 \mathrm{MeV}$, respectively. They are considered as $\langle s \bar{s}>$ condensates and interact only with the $s$-quarks in the baryons. This picture is consistent with the chiral models [8,9]. We fixed the total strangeness density $\rho_{S}$ to 0 by introducing an ideal gas of $K$ and $K^{*}$ mesons where $m_{K}=495 \mathrm{MeV}$ and $m_{K^{*}}=$ $892 \mathrm{MeV}$. The contribution of other $K$ mesons was found to be negligible. It is supposed in the ideal gas limit that the Kaons do not interact with the mean fields $\zeta$ and $\phi$. The extension to the case that the Kaons interact with the $\zeta$ and $\phi$ fields will be considered in a future work.

We first solve Eqs. (18), (19), (20) and (21) self consistently for given values of temperature $T$ and densities $\rho_{B}$ and $\rho_{S}$ to determine the baryonic and strangeness chemical potentials $\mu_{B}$ and $\mu_{S}$, respectively. These constraints are given in terms of the effective baryon masses $M_{i}^{*}$ which depend on the bag radii $R_{i}$, the quark chemical potentials $\mu_{q}^{i}$ and the mean fields. For given values of the scalar fields $\sigma, \zeta$ and vector fields $\omega, \phi$, the quark chemical potential and bag radius of species $i$ are obtained using the self consistency conditions Eqs. (7) and (14), respectively. The pressure is evaluated for specific values of temperature $T$ and chemical potentials $\left(\mu_{B}, \mu_{S}\right)$ which now become input parameters. We then determine the values of $\sigma$ and $\zeta$ by using the extermization conditions as given in Eq. (28). These constraints take into account the coupling of the quark with the scalar mean fields in the frame of the point like Dirac equation exactly [7,12].

The dependence of the baryon effective masses $M_{N, \Lambda, \Sigma, \Xi}^{*}$ on the total baryonic density $\rho_{B}$ and temperature is shown in Fig. 1 where it is seen that the baryon masses decrease with baryonic density except at the highest temperatures where the effective masses become almost density-independent. Moreover, for a given baryonic density $\rho_{B}$ it is seen that as the temperature is increased the mass $M_{i}^{*}$ of species $i$ first increases slightly up to about $T=150$ $\mathrm{MeV}$ and then decreases rather rapidly for $T>150 \mathrm{MeV}$. These results are displayed in a different manner in Fig. 2 which plots the baryon masses $M_{i}^{*}$ as a function of $T$ for $\rho_{B}=0$ $\mathrm{fm}^{-3}$ and $\rho_{B_{0}}=0.17 \mathrm{fm}^{-3}$. It is seen that the effective baryon masses $M_{N, \Lambda, \Sigma, \Xi}^{*}$ with $\rho_{B_{0}}=0.17 \mathrm{fm}^{-3}$ are less than those with zero baryonic density. Moreover, the effective baryonic masses $M_{N, \Lambda, \Sigma, \Xi}^{*}$ increase only slightly, if at all, with temperature up to about $T=150 \mathrm{MeV}$ beyond which they decrease rapidly. This behaviour is qualitatively similar to our earlier results for normal nuclear matter [23, 18, 19] where the rapid decrease in the nucleon's effective mass was, however, found to start at rather higher temperatures $T>200$ $\mathrm{MeV}$. This rapid decrease of $M_{i}^{*}$ with increasing temperature resembles a phase transition at high temperatures and low density, when the system becomes a dilute gas of baryons in a sea of baryon-antibaryon pairs [23].

In Fig. 3, we display the scalar mean fields $\sigma$ and $\zeta$ as functions of the total baryonic density $\rho_{B}$ for various temperatures. It is seen that the value of $\sigma$ initially decreases with increasing temperature for temperatures less than $150 \mathrm{MeV}$. The scalar mean field $\zeta$ is almost negligible for such low temperatures. However, as the temperature reaches $150 \mathrm{MeV}$ there are indications of an increase in $\sigma$ at low baryon densities where it attains a nonzero 
value at $\rho_{B}=0$. For still higher temperatures, the situation is more dramatic with the value of $\sigma$ increasing with temperature for all values of $\rho_{B}$. This is also qualitatively similar to our earlier results for $S U(2)$ nuclear matter [18,19]. Furthermore, the scalar mean field $\zeta$ becomes important for $T>150 \mathrm{MeV}$ and also increases with temperature for all values of $\rho_{B}$. An interesting new feature here is that the scalar mean fields $\sigma$ and $\zeta$ tend to take almost constant values irrespective of density at high temperatures which was not seen in our earlier calculations for $S U(2)$ nuclear matter even at temperatures as high $240 \mathrm{MeV}$. Fig. 4 displays $\sigma$ and $\zeta$ versus $T$ for $\rho_{B}=0$ and $\rho_{B 0}=0.17 \mathrm{fm}^{-3}$. It is seen that the $\sigma$ field has a nonzero (and almost constant) value only at the higher density until the temperature reaches about $T=150 \mathrm{MeV}$ when a rapid increase sets in at both densities. The increase at zero density is actually more dramatic and the $\sigma$ field rapidly attains values equal to those occuring at $\rho_{B_{0}}=0.17 \mathrm{fm}^{-3}$. This is another indication of a phase transition to a system of baryon-antibaryon pairs. The behaviour of the $\zeta$ field is qualitatively similar except that its value is negligible at both densities for temperatures less than about $T=150 \mathrm{MeV}$.

In Fig. 5, we display the baryonic density dependence of the bag parameters for $N, \Lambda, \Sigma, \Xi$ for different values of the temperature. For each baryon, the bag parameter increases with temperature for temperatures less than $150 \mathrm{MeV}$. However, the situation is completely reversed after the phase transition takes place. For temperatures $T>150 \mathrm{MeV}$ the bag parameters display a dramatic decrease with temperature for all densities. This can be seen more clearly in Fig. 6 which displays $B_{i}$ vs $T$ for $\rho_{B}=0 \mathrm{fm}^{-3}$ and $\rho_{B_{0}}=0.17 \mathrm{fm}^{-3}$. The bag parameters are almost constant until the temperature exceeds $T=150 \mathrm{MeV}$ when they start to decrease rapidly. This indicates the onset of quark deconfinement above the critical temperature: at high enough temperature and/or baryon density there is a phase transition from the baryon-meson phase to the quark-gluon phase. This behaviour is also in qualitative agreement with our earlier results for $S U(2)$ nuclear matter [18] except that the decrease here is more dramatic indicating that the phase transition is much stronger than in ordinary nuclear matter. Our results are also comparable to those obtained from lattice QCD calculations which have so far only explored the zero baryon density axis of the phase diagram in a meaningful way. The lattice results with 2 light quark flavors, indicate that the transition from hadronic matter to a quark-gluon plasma occurs at a temperature $T \approx 140$ $\mathrm{MeV}$ and that for low densities it may not be a phase transition at all but what is called a rapid crossover [24].

Fig. 7 displays the relative abundance $\rho_{i} / \rho_{B}$ for each baryon species. At low temperatures the nucleons $N$ are almost the only constituents. However, the contribution of the hyperons starts to be noticeable when the temperature reaches $100 \mathrm{MeV}$ and becomes more important when the temperature is increased to $T>150 \mathrm{MeV}$. At temperatures $T>200$ $\mathrm{MeV}$ the contribution of the nucleons falls down to about half the total baryonic density. This can also be seen in Fig. 8 where we display the ratio of the baryonic strangeness density to the total baryonic density. As mentioned earlier, we keep the net strangeness of the system fixed to zero by introducing Kaons so that $\rho_{S}=\rho_{S}^{\text {Baryons }}+\rho_{S}^{\text {Mesons }}=0$. It is seen that the net strangeness of the baryons $\rho_{S}^{\text {Baryons }}$ is small at low temperatures $T<150 \mathrm{MeV}$ (note the logarithmic scale). However, as the temperature increases the net strangeness of the baryon octet becomes significant. For $T>150 \mathrm{MeV}$, the ratio $\rho_{S}^{\text {Baryons }} / \rho_{B}$ becomes of order one. Therefore, the $K$-meson plays a significant role at high temperatures and probably also in the phase transition. 
In Fig. 9, we display the relative abundances of the various baryon species $\rho_{i} / \rho_{B}$ versus temperature at normal nuclear matter density $\rho_{B_{0}}=0.17 \mathrm{fm}^{-3}$. It is seen that the the nucleons are dominant at low temperatures and their relative abundance $\rho_{N} / \rho_{B}$ decreases very slowly at first and deviates very little from 1 until the temperature reaches about 100 $\mathrm{MeV}$ when $\rho_{N} / \rho_{B}$ starts to decrease rapidly and becomes negligibly small for temperatures larger than about $280 \mathrm{MeV}$. On the other hand, the abundance of the hyperons is negligible at low temperatures and increases significantly as the temperature is increased beyond $T=100$ $\mathrm{MeV}$. They become more abundant than the nucleons for temperatures larger than $200 \mathrm{MeV}$. The relative abundance of hyperons in high energy heavy ion collisions can therefore be used as a simple thermometer to measure the temperature of the hot nuclear matter produced in the reaction and probably to study the phase transition to the quark gluon plasma.

In conclusion, we have generalized the MQMC model to the case of hot hypernuclear matter by introducing two new meson fields that couple to the strange quarks and using $S U(6)$ symmetry to relate the coupling constants. The results are qualitatively similar to those obtained for $S U(2)$ nuclear matter including the onset of quark deconfinement. It

is observed, however, that in this model quark deconfinement in $S U(3)$ (or hypernuclear) matter is much stronger than in $S U(2)$ (or normal) nuclear matter and that hyperons become more abundant than nucleons at high temperatures $T>200 \mathrm{MeV}$. An ideal gas of Kaons was introduced to keep zero net strangeness density. The interaction of the Kaons with the meson fields will be considered in a future work.

\section{ACKNOWLEDGMENTS}

Financial support by the Deutsche Forschungsgemeinschaft through the grant GR 243/51-1 is gratefully acknowledged. 


\section{REFERENCES}

[1] J. D. Walecka, Ann. of Phys. 83, 491 (1974); Phys. Lett. B 59, 109 (1975).

[2] B. D. Serot and J. D. Walecka, Adv. Nucl. Phys. 16, 1 (1986).

[3] B. D. Serot and J. D. Walecka, nucl-th/9701058.

[4] M. Arneodo, Phys. Rep. 240, 301 (1994).

[5] P. A. M. Guichon, Phys. Lett. B 200, 235 (1988).

[6] P. G. Blunden and G. A. Miller, Phys. Rev. C 54, 359 (1996).

[7] K. Saito and A. W. Thomas, Phys. Lett. B 327, 9 (1994).

[8] P. Papazoglou, S. Schramm, J. Schaffner-Bielich, H. Stöcker, and W. Greiner, Phys. Rev. C 57, 2576 (1998).

[9] P. Papazoglou, D. Zschiesche, S. Schramm, J. Schaffner-Bielich, H. Stöcker, and W. Greiner, Phys. Rev. C 59, 411 (1999).

[10] K. Tsushima, K. Saito, J. Haidenbauer, and A. W. Thomas, Nucl. Phys. A 630, 691 (1998).

[11] K. Saito, K. Tsushima, and A. W. Thomas, Phys. Rev. C 55, 2637 (1997).

[12] X. Jin and B. K. Jennings, Phys. Rev. C 54, 1427 (1996); Phys. Lett. B 374, 13 (1996).

[13] X. Jin and B. K. Jennings, Phys. Rev. C 55, 1567 (1997); H. Müller and B. K. Jennings, Nucl. Phys. A 626, 966 (1997); H. Müller, Phys. Rev. C 57, 1974 (1998).

[14] T. D. Cohen, R. J. Furnstahl, D. K. Griegel, and X. Jin, Prog. Part. Nucl. Phys. 35, 221 (1995).

[15] M. K. Banerjee, Phys. Rev. C 45, 1357 (1992); V. K. Mishra, Phys. Rev. C 46, 1143 (1992); E. Naar and M. C. Birse, J. Phys. G. 19, 555 (1993).

[16] H. Q. Song and R. K. Su, Phys. lett. B 358, 179 (1995).

[17] P. K. Panda, A. Mishra, J. M. Eisenberg, and W. Greiner, Phys. Rev. C 56, 3134 (1997).

[18] I. Zakout and H. R. Jaqaman, Phys. Rev. C 59, 962 (1999).

[19] I. Zakout and H. R. Jaqaman, Phys. Rev. C 59, 968 (1999).

[20] J. Schaffner, C. B. Dover, A. Gal, C. Greiner, and H. Stöcker, Phys. Rev. Lett. 71, 1328 (1993); J. Schaffner-Bielich, C. Greiner, A. Diener, and H. Stöcker, Phys. Rev. C 55, 3038 (1997). .

[21] H. Müller, nucl-th/ 9810055 .

[22] S. Fleck, W. Bentz, K. Shimizu, and K. Yazaki, Nucl. Phys. A510, 731 (1990).

[23] R. J. Furnstahl and B. D. Serot, Phys. Rev. C 41, 262 (1990).

[24] S. Gottlieb, U. M. Heller, A. D. Kennedy, S. Kim, J. B. Kogut, C. Liu, R. L. Renken, D. K. Sinclair, R. L. Sugar, D. Toussaint, and K. C. Wang, Phys. Rev. D 55, 6852 (1997); C. Bernard, T. Blum, C. DeTar, S. Gottlieb, K. Rummukainen, U. M. Heller, J. E. Hetrick, D. Toussaint, L. Karkkainen, R. L. Sugar, and M. Wingate, Phys. Rev. D 55, 6861 (1997). 


\section{FIGURES}

Fig. 1
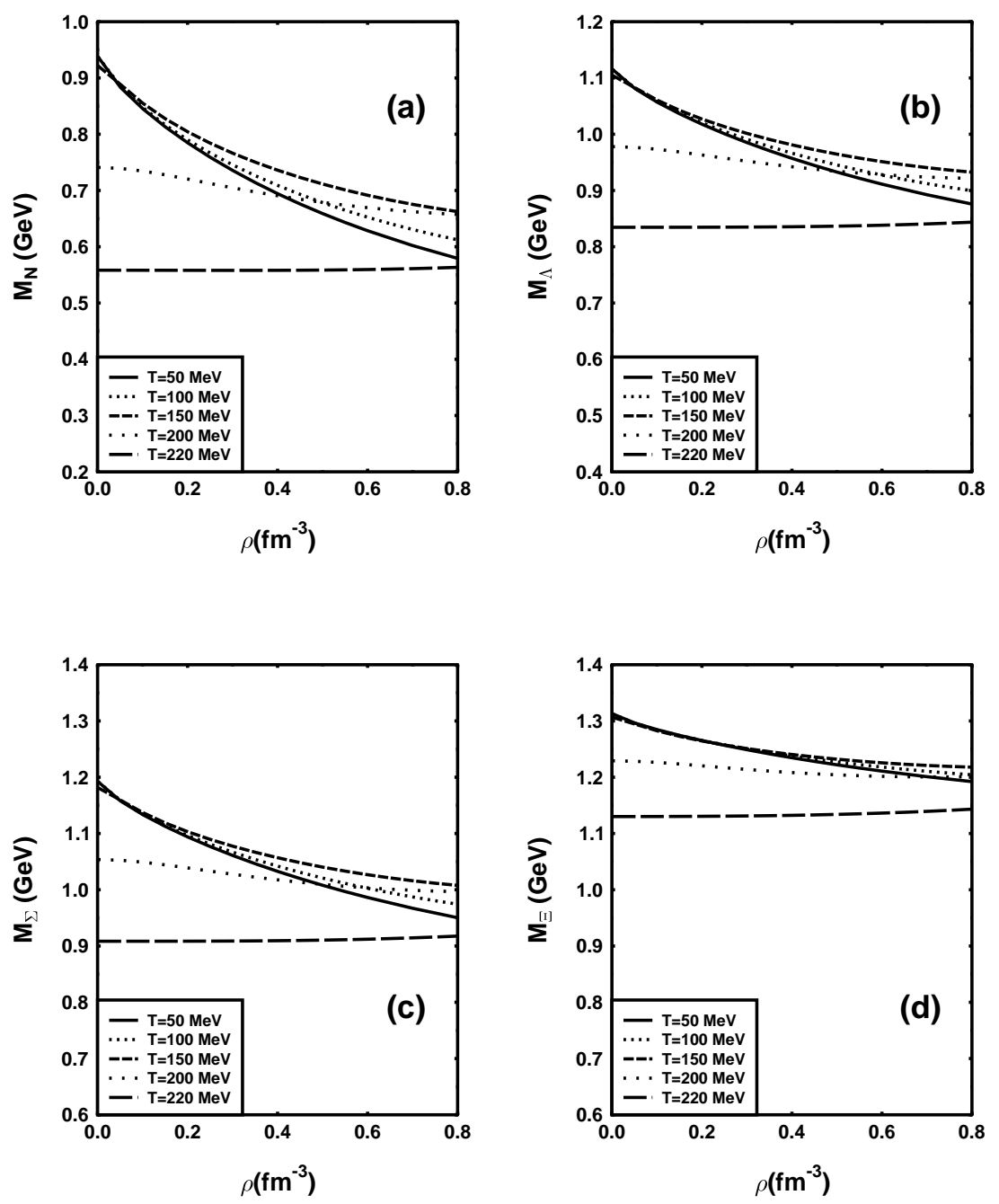

FIG. 1. The effective baryon masses $M_{i}^{*}$ in hypernuclear matter as a function of the total baryonic density $\rho_{B}$ for different values of temperature. 
Fig. 2

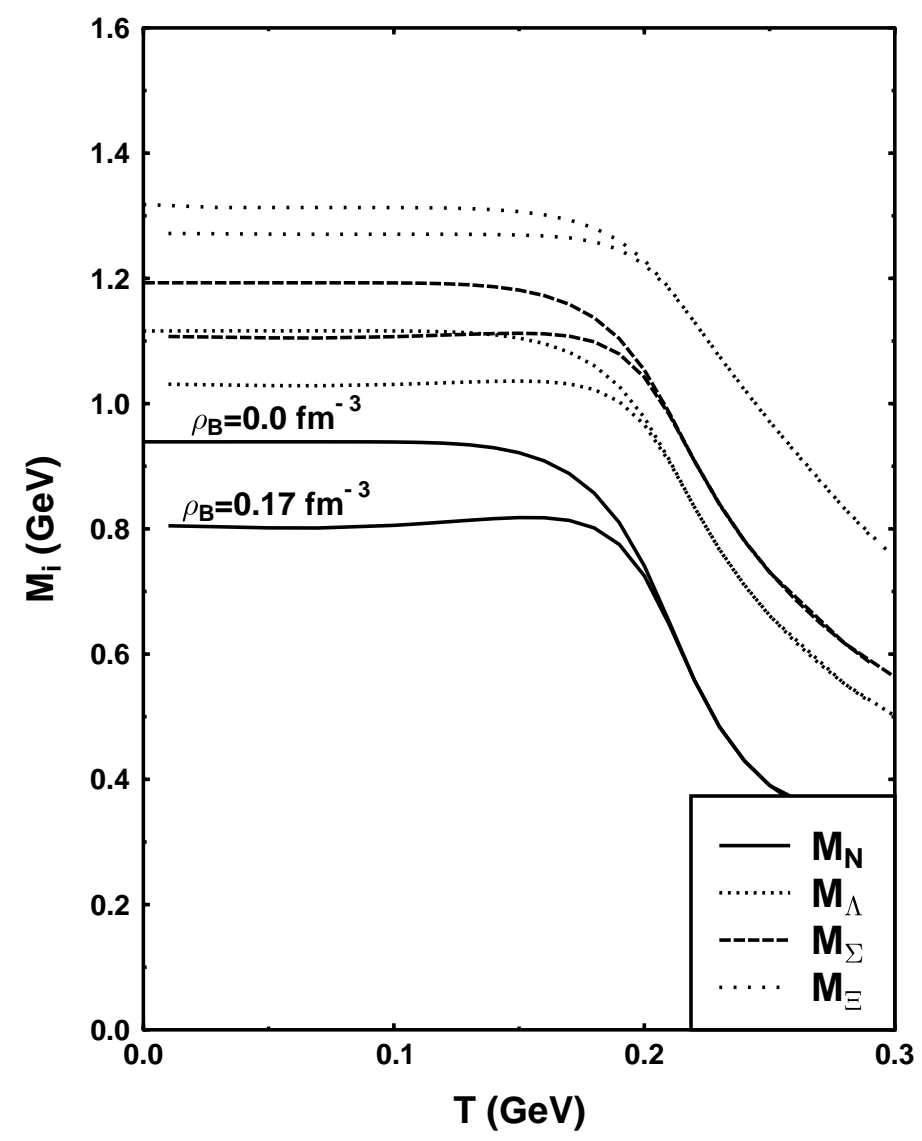

FIG. 2. The effective baryon masses $M_{i}^{*}$ in hypernuclear matter as a function of temperature for $\rho_{B}=0$ and $\rho_{B}=0.17 \mathrm{fm}^{-3}$. 
Fig. 3

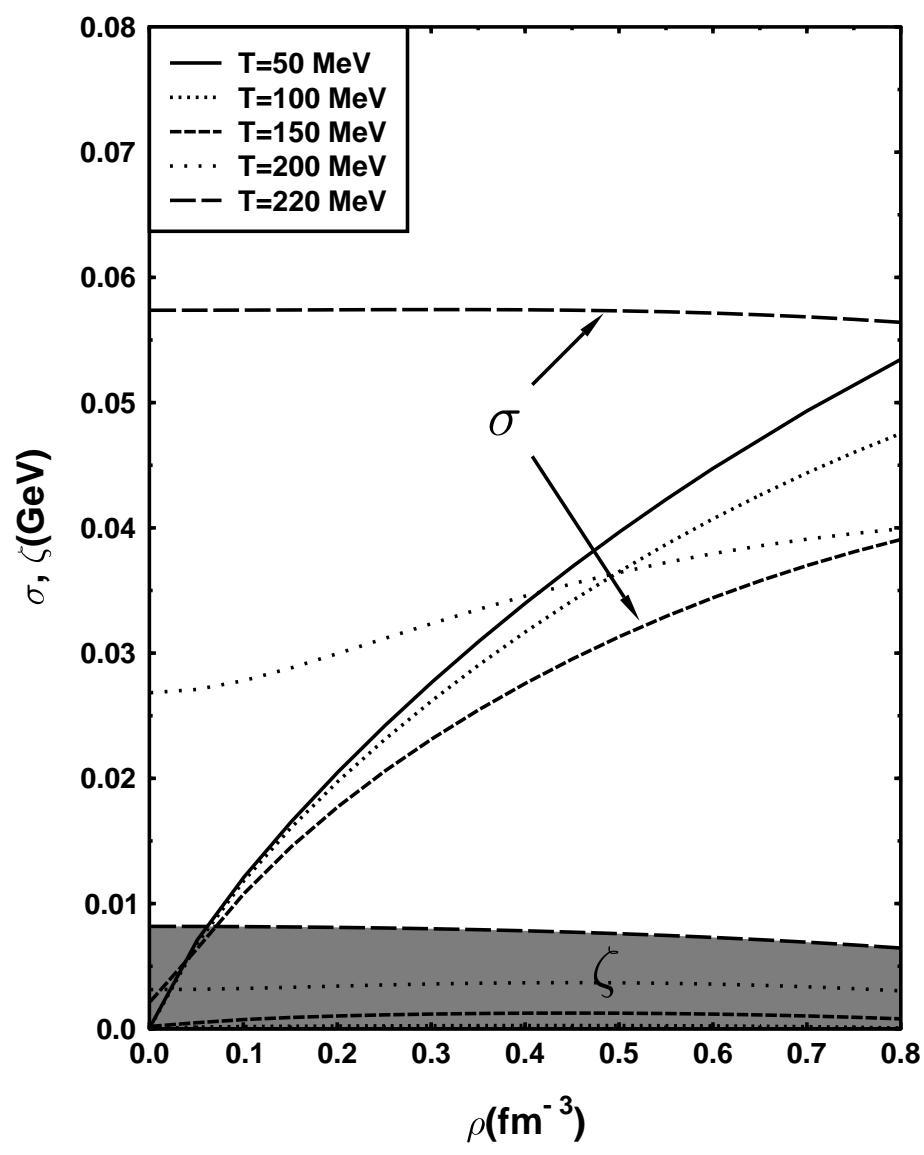

FIG. 3. The mean scalar fields $\sigma, \zeta$ as a function of the total baryonic density $\rho_{B}$ for different values of temperatures. 
Fig. 4

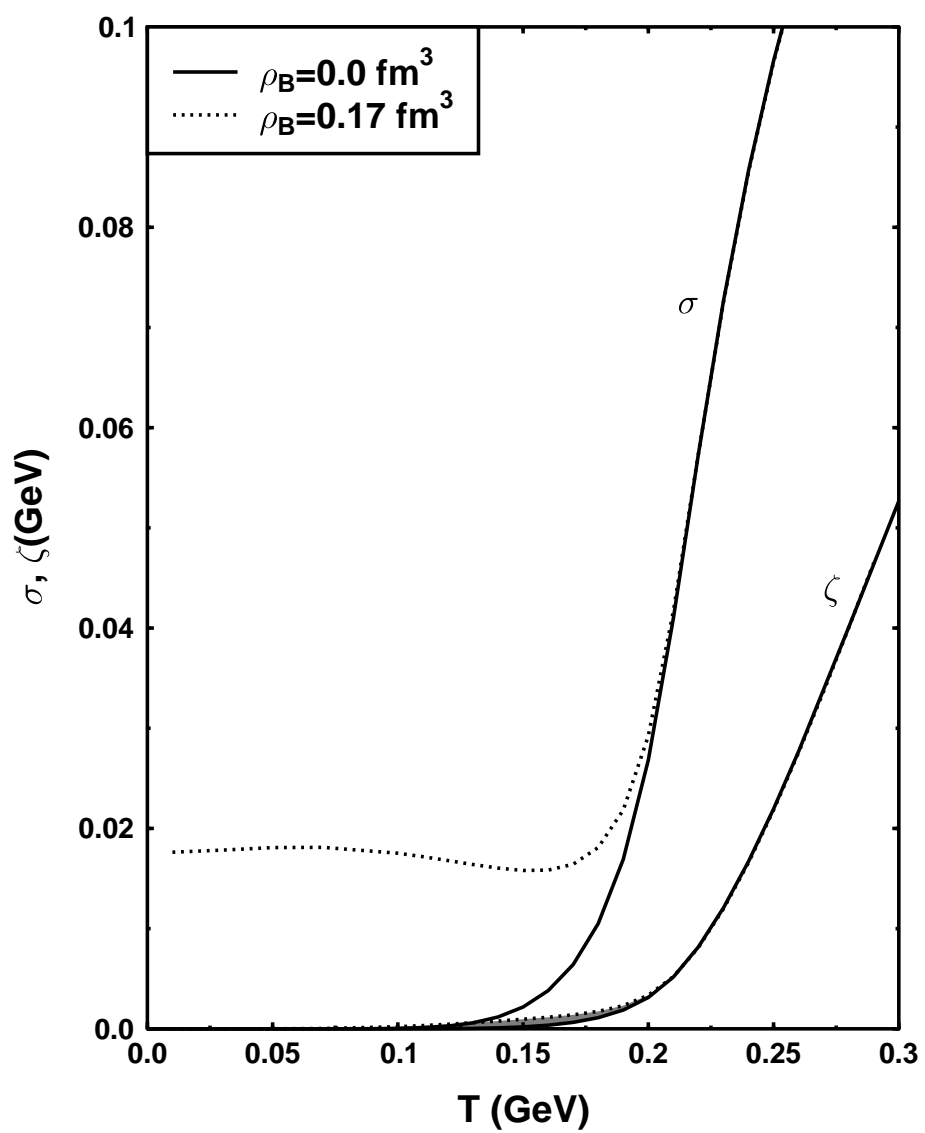

FIG. 4. The mean scalar fields $\sigma, \zeta$ versus the temperature for $\rho_{B}=0$ and $\rho_{B}=0.17 \mathrm{fm}^{-3}$. 
Fig. 5
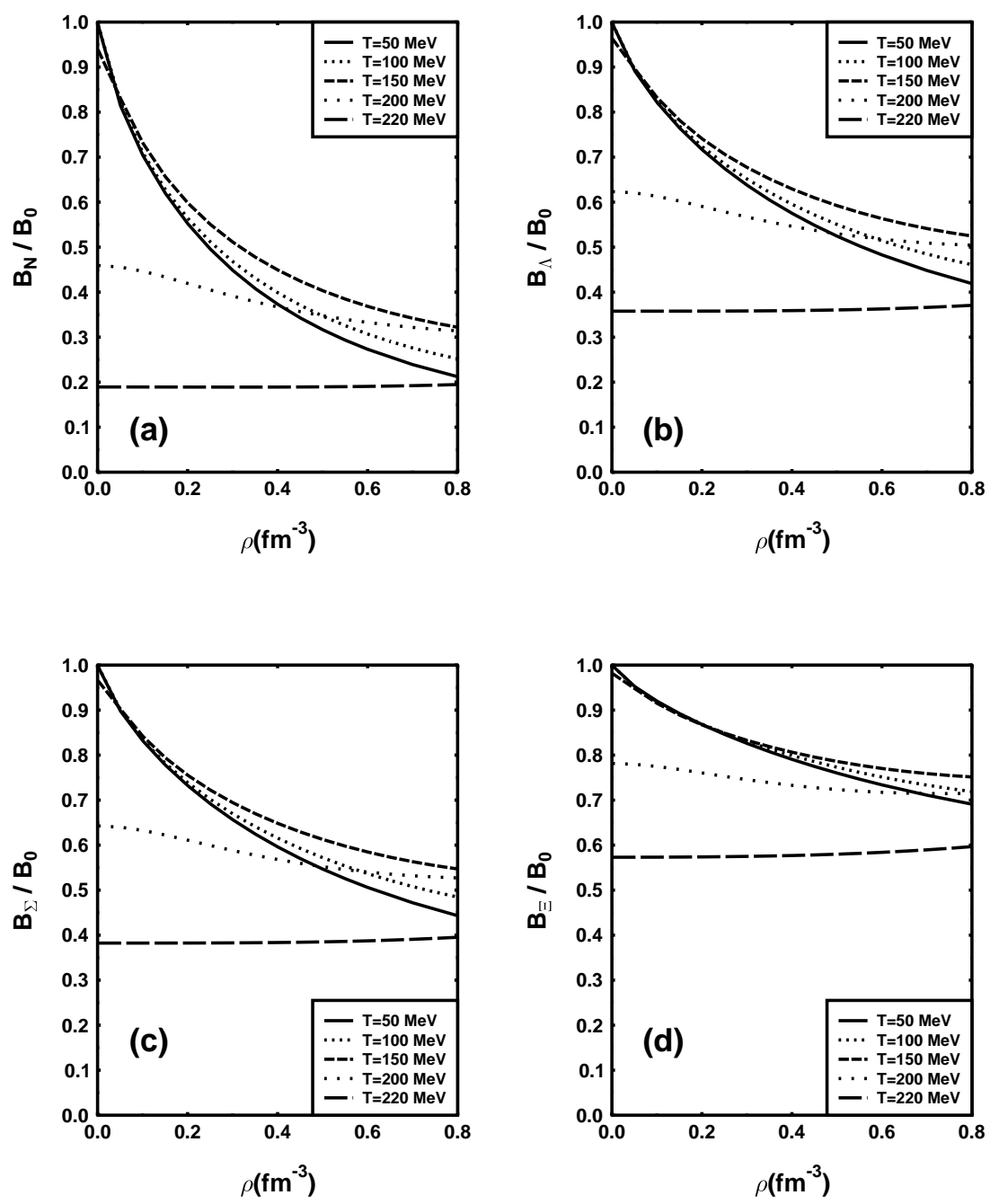

FIG. 5. The bag parameters $B_{i}$ of the nucleon and hyperons versus the total baryonic density $\rho_{B}$ for different values of temperature. 
Fig. 6

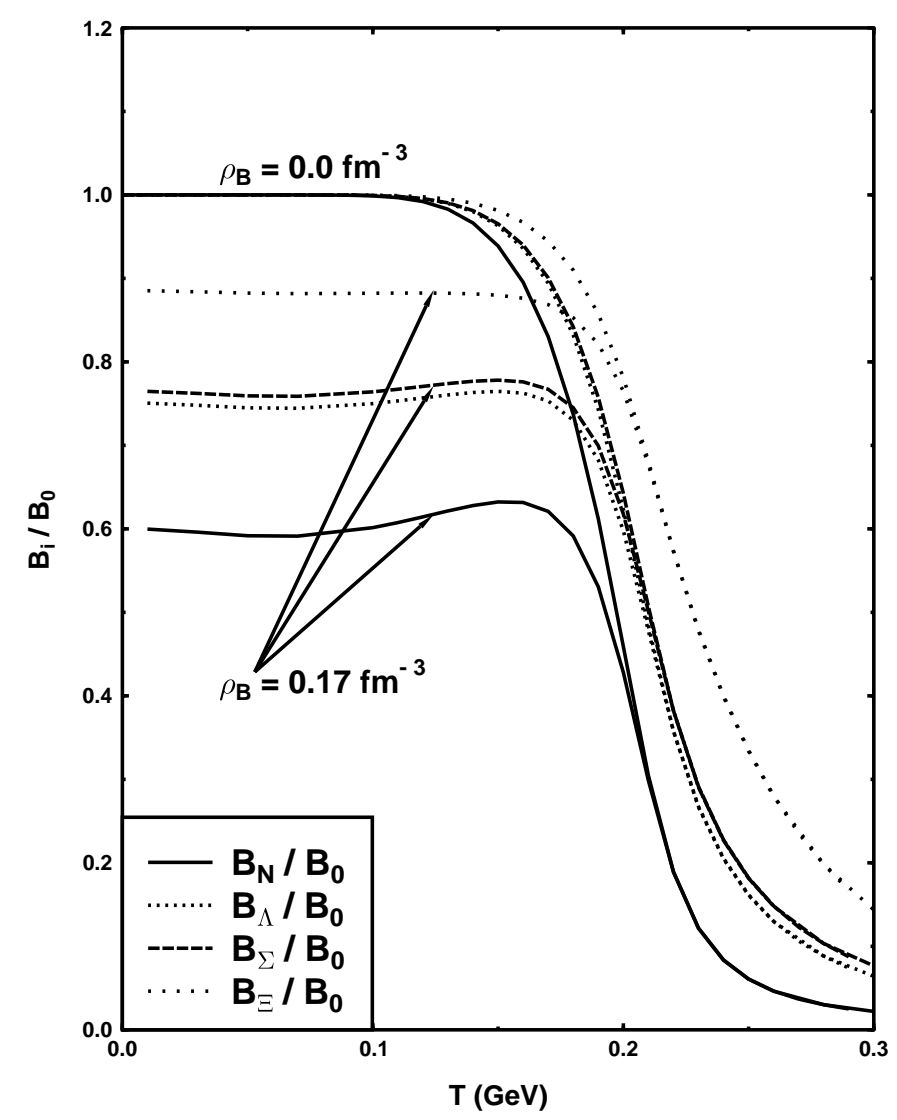

FIG. 6. The bag parameters $B_{i}$ of the nucleon and hyperons versus the temperature $T$ for $\rho_{B}=0$ and $\rho_{B}=0.17 \mathrm{fm}^{-3}$. 
Fig. 7
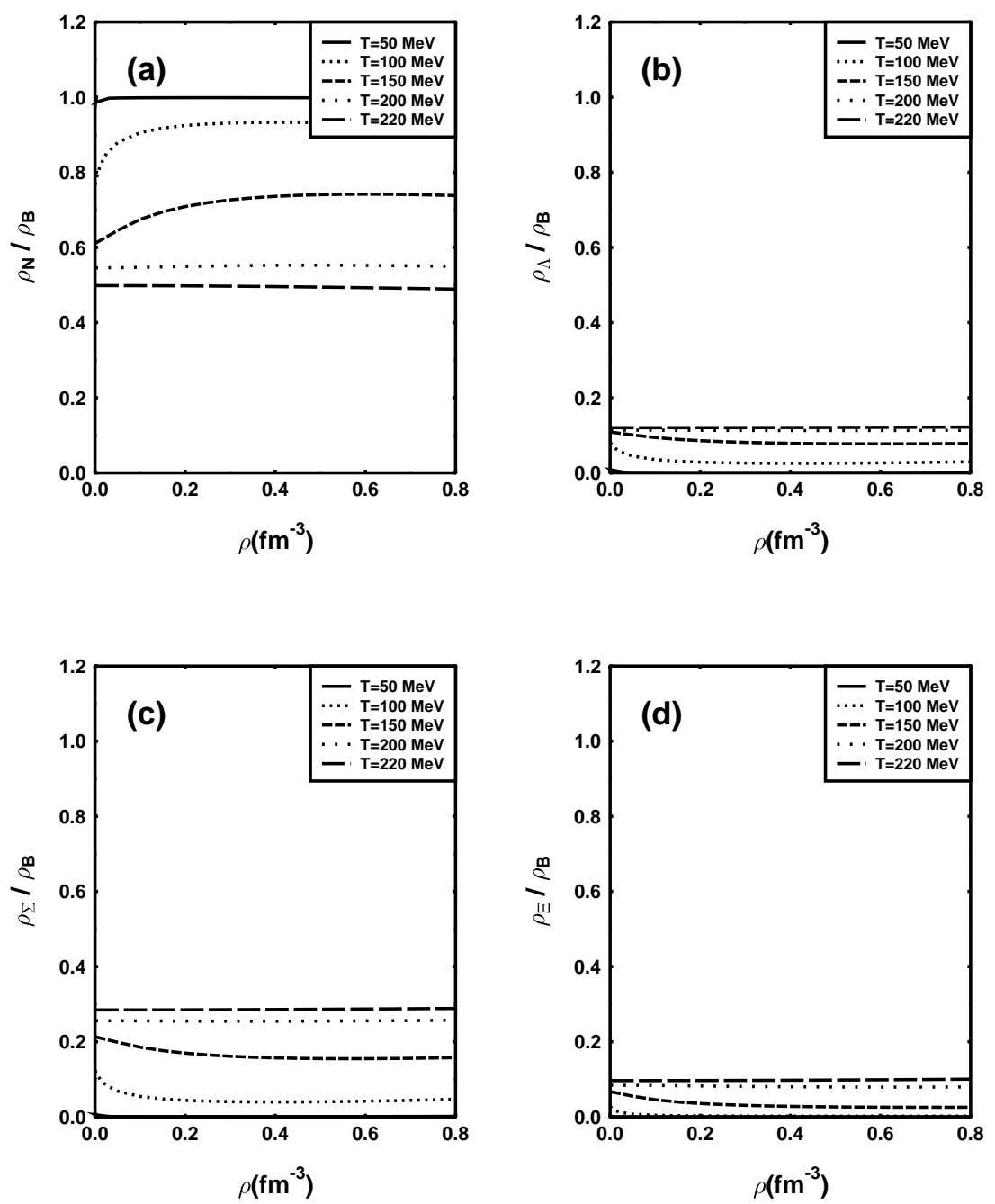

FIG. 7. The relative abundance of baryon species $i, \frac{\rho_{i}}{\rho_{B}}$, versus the total baryonic density $\rho_{B}$ for different temperature. 
Fig. 8

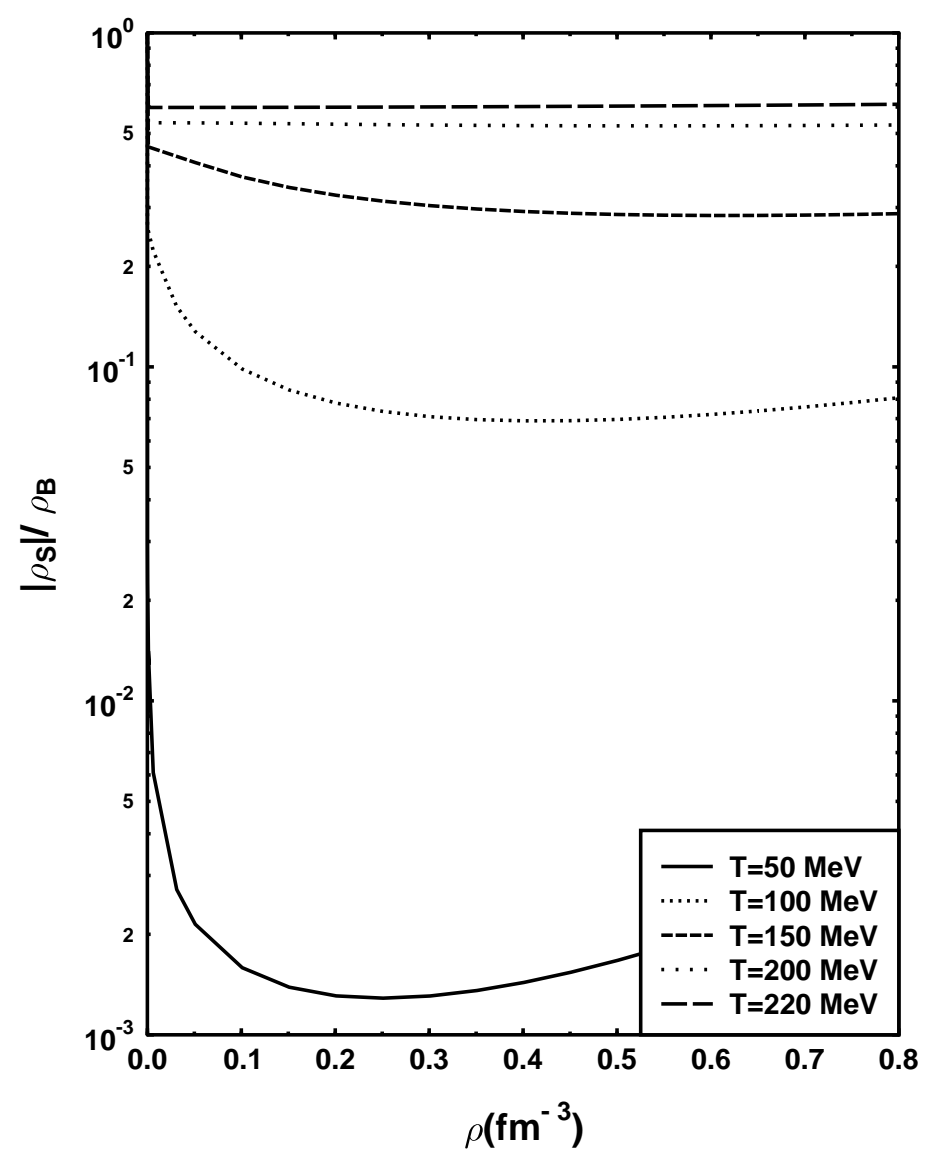

FIG. 8. The absolute value of the ratio of the baryonic stangeness density to the total baryonic density $\rho_{S} / \rho_{B}$ as a function of the total baryonic density $\rho_{B}$ for various temperatures. 
Fig. 9

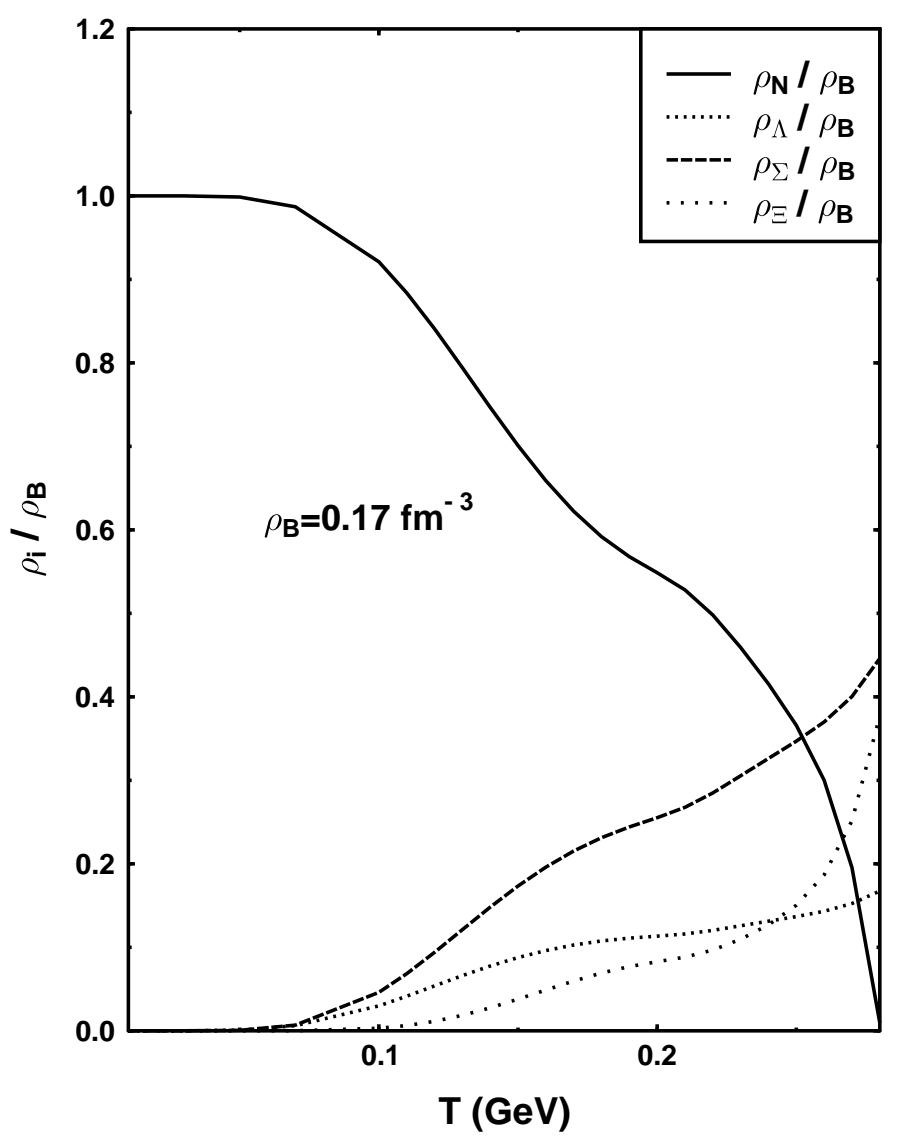

FIG. 9. The relative abundance of baryon species $i, \frac{\rho_{i}}{\rho_{B}}$, versus temperature $T$ for $\rho_{B}=0.17$ $\mathrm{fm}^{-3}$. 